\title{
Cationic metal-organic frameworks as an efficient adsorbent for the removal of 2,4-dichlorophenoxyacetic acid from aqueous solutions
}

\author{
Gege $\mathrm{Wu}^{\mathrm{a}}$, Jiping $\mathrm{Ma}^{\mathrm{a}}{ }^{\mathrm{a} * *}$, Shuang $\mathrm{Li}^{\mathrm{a}}$, Shasha Wang ${ }^{\mathrm{a}}$, Bo Jiang ${ }^{\mathrm{a}}$, Siyi Luo ${ }^{\mathrm{a}}$, Jinhua $\mathrm{Li}^{\mathrm{b}, \mathrm{d}}$, \\ Xiaoyan Wang ${ }^{\mathrm{b}, \mathrm{c}}$, Yafeng Guan ${ }^{\mathrm{e}}$, Lingxin Chen ${ }^{\mathrm{b}, \mathrm{c}, \mathrm{d}, *}$

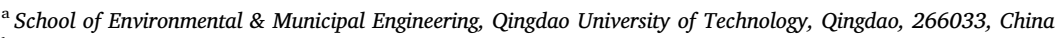 \\ ${ }^{\mathrm{b}}$ CAS Key Laboratory of Coastal Environmental Processes and Ecological Remediation, Research Center for Coastal Environmental Engineering and Technology, Yantai \\ Institute of Coastal Zone Research, Chinese Academy of Sciences, Yantai, 264003, China \\ ${ }^{\mathrm{c}}$ School of Pharmacy, Binzhou Medical University, Yantai, 264003, China \\ ${ }^{\mathrm{d}}$ Shandong Key Laboratory of Coastal Environmental Processes, Yantai Institute of Coastal Zone Research, Chinese Academy of Sciences, Yantai, 264003, China \\ ${ }^{\mathrm{e}}$ CAS Key Laboratory of Separation Sciences for Analytical Chemistry, Dalian Institute of Chemical Physics, Chinese Academy of Sciences, Dalian, 116023, China
}

\section{A R T I C L E I N F O}

\section{Keywords:}

Ion exchange

Metal-organic frameworks (MOFs)

2,4-Dichlorophenoxyacetic acid (2,4-D)

Adsorptive removal

Water sample

\begin{abstract}
A B S T R A C T
Metal-organic frameworks (MOFs) material with high surface area, good chemical stability and multi-functionality, has become an emerging adsorbent for water treatment. A novel kind of quaternary amine anionicexchange MOFs UiO-66 namely UiO-66- $\mathrm{NMe}_{3}{ }^{+}$was firstly synthesized for adsorptive removal of a widely used toxic herbicide 2,4-dichlorophenoxyacetic acid (2,4-D) from aqueous solutions. The well-prepared UiO-66$\mathrm{NMe}_{3}{ }^{+}$MOFs were fully characterized, and then the main parameters affecting the adsorption process including solution $\mathrm{pH}$, adsorbent dosage and coexisting anions were systematically investigated. The maximum adsorption capacity of UiO-66- $\mathrm{NMe}_{3}{ }^{+}$toward 2,4-D reached as high as $279 \mathrm{mg} \mathrm{g}^{-1}$, much higher than that of pristine UiO66 and aminated UiO-66. The adsorption mechanism could be attributed to the electrostatic interactions efficiently enhanced by the functionalization of quaternary amine groups, combining with the $\pi-\pi$ conjugations between the linkers in MOFs and 2,4-D molecules, leading to the better adsorption performance of UiO-66$\mathrm{NMe}_{3}{ }^{+}$. Additionally, the UiO-66- $\mathrm{NMe}_{3}{ }^{+}$could be well regenerated by simple solvent washing and exhibited a slight decline of adsorption capacity after seven successive recycle. Furthermore, satisfactory adsorption capacity and reusability of the MOFs in environmental water samples were attained. Comparing with reported activated carbon and resin materials, the UiO-66- $\mathrm{NMe}_{3}{ }^{+}$MOFs possessed higher adsorption capacity and shorter equilibrium time, as well as good reusability and practicality. The developed ion-exchange functionalized MOFs provided an ideal alternative for efficient adsorptive-removal of 2,4-D from complicated aqueous environment.
\end{abstract}

\section{Introduction}

Phenoxy acid herbicides are widely used as plant growth regulator on grass and broadleaf weeds. Among these herbicides, 2,4-dichlorophenoxyacetic acid (2,4-D) exhibits excellent selectivity with relatively low cost, and has become one of the most widely used phenoxy acid herbicides (Lu et al., 2012). However, 2,4-D is also an endocrine disruptor showing high toxicity to kidney, liver and central nervous system, and the International Agency for Research on Cancer has classified it as a potential human carcinogen and mutagen (DeryloMarczewska et al., 2010). As an ionizable herbicide, the excessive residues of 2,4-D are usually weakly adsorbed onto soil components
(Mantilla et al., 2009) and can easily move into natural environment water bodies because of its low $\mathrm{p} K_{\mathrm{a}}$. So far, the extensive use of 2,4-D has made it be frequently detected in surface water and groundwater (Ignatowicz, 2009). When the concentration of 2,4-D rises to $10-30 \mathrm{mg} \mathrm{L}^{-1}$ in natural waters, human health would be affected (Aaron and Oturan, 2001). For this reason, it's necessary to develop effective materials and technologies to eliminate 2,4-D from aqueous media to avoid its accumulation in aquatic environment.

Nowadays, different kinds of treatment methods have been developed to remove 2,4-D from aqueous solutions, such as photocatalytic degradation (Echavia et al., 2009), Fenton processes (Katsumata et al., 2011), biological treatment (Cycon et al., 2011) and nanofiltration

\footnotetext{
${ }^{*}$ Corresponding author. CAS Key Laboratory of Coastal Environmental Processes and Ecological Remediation, Research Center for Coastal Environmental Engineering and Technology, Yantai Institute of Coastal Zone Research, Chinese Academy of Sciences, Yantai, 264003, China.

** Corresponding author. School of Environmental \& Municipal Engineering, Qingdao University of Technology, Qingdao, 266033, China.

E-mail addresses: majiping2012@163.com (J. Ma), lxchen@yic.ac.cn (L. Chen).
} 
membranes (Ehtash et al., 2014). Compared to these techniques, adsorption method possesses the advantages such as simple operation, low cost, few toxic byproducts and high removal efficiency and has been widely used for the removal of pollutants from aqueous solutions ( $\mathrm{Lu}$ et al., 2017). The removal efficiency of adsorption is directly dependent on the properties of adsorbents. Activated carbon is the most widely used material for the removal of organic micropollutants, while the properties of 2,4-D make it difficult to be removed by traditional carbon adsorbents (Corwin and Summers, 2012). Therefore, several functionalized materials have been investigated as the adsorbents for the removal of 2,4-D from water, e.g., activated carbon chemically activated by $\mathrm{H}_{3} \mathrm{PO}_{4}$ (Njoku and Hameed, 2011), biopolymers of chitin and chitosan (Harmoudi et al., 2014), and Pd/Fe functionalized magnetic MWCNTs nanoparticles (Xu et al., 2016).

Metal-organic frameworks (MOFs) are a new class of porous material built by various transition metal ions and organic ligands. They have attracted a great deal of interest due to their unique structures and properties, such as high surface area, good thermal and chemical stability, and multi-functionality (Wu et al., 2018; Ma et al., 2016). The wide applications of MOFs have been demonstrated in gas storage (Rosi et al., 2003; Yang et al., 2017a), catalysis (Rostamnia et al., 2016; Hu et al., 2014a), sensing (Yang et al., 2013; Zhang et al., 2012), and adsorption removal of pollutants (Hasan and Jhung, 2015; Dias and Camille, 2015). The electrically neutral nature of MOFs makes them exhibit relatively high adsorption efficiency for weak-polar substances such as polycyclic aromatic hydrocarbons (PAHs) (Xie et al., 2015; Hu et al., 2014b), benzene series (He et al., 2013) and heterocyclic pesticides (Ma et al., 2018). Furthermore, the functionalization of MOFs will improve their applicability in polar atmosphere (Burtch et al., 2014; De et al., 2012). Therefore, the functionalization process is a promising approach to extend the applications of MOFs in water treatment. Ionexchange MOFs (iMOFs) are novel materials by combining porous MOFs with ion exchangers. Compared with conventional ion exchangers, several iMOFs have exhibited better chemical and thermal stability (Kumar et al., 2017). Due to various porous structures and binding groups, the functionalization of MOFs with ion-exchange groups shows higher selectivity toward targeted ionic compounds. For example, Liu et al. (2015) used two modification methods to functionalize MIL-101(Cr) with anionic exchangers, and applied them as the adsorbents for the removal of perfluorooctanoic acid from aqueous solution. Zhang et al. (2017) used $\mathrm{Fe}_{3} \mathrm{O}_{4} @ \mathrm{SiO}_{2}$ combined quaternary amine functionalized MIL-101(Cr) for magnetic solid-phase extraction of azide in sartan drugs. The experimental results indicated that iMOFs tended to exhibit remarkable enhancement in adsorption efficiency compared to unmodified MOFs. However, the investigation on the application of iMOFs is just in the opening stage, and the iMOFs haven't been extensively applied in environmental contaminant removal.

As a zirconium-based MOF, UiO-66 possesses large surface area, exceptional thermostability and good chemical resistance, and has been used for the removal of PAHs (Gao et al., 2016), sulfachloropyridazine (Azha et al., 2017), and nitrophenol (Yang et al., 2017b). And, UiO-66 based iMOFs have been used for the removal of hexavalent chromium (Rapti et al., 2016) and perrhenate (Banerjee et al., 2016) from water. However, to the best of our knowledge, quaternary amine functionalized UiO-66 based iMOFs have not been reported up to now.

Therefore, inspired by the above researches about the preparation and applications of MOFs, herein, we synthesized the quaternary amine anionic-exchange MOF UiO-66 (UiO-66- $\mathrm{NMe}_{3}{ }^{+}$) for adsorptive removal of 2,4-D. The well-prepared UiO-66- $\mathrm{NMe}_{3}{ }^{+}$was thoroughly characterized. Several main influence factors on adsorption efficiency such as the adsorbent dosage, initial solution $\mathrm{pH}$ and coexisting anions were examined in detail. In addition, the adsorption properties and the reusability of the UiO-66- $\mathrm{NMe}_{3}{ }^{+}$were investigated, as well as the adsorption capacity and reusability in three environmental water samples were evaluated. Furthermore, the possible adsorption mechanism was explored.

\section{Materials and methods}

\subsection{Reagents and materials}

All reagents were of at least analytical grade. 2,4-D (as shown in Fig. $\mathrm{S} 1$ in electronic supporting information (ESI)) and 2-amino-terephthalic acid were purchased from Shanghai Aladdin Chemistry Co., Ltd. (Shanghai, China). Zirconium chloride $\left(\mathrm{ZrCl}_{4}\right)$ was acquired from Sinopharm Chemical Reagent Co. Ltd. (Shanghai, China), and methyl triflate was purchased from Jiuding Chemical Reagent Limited Company (Shanghai, China). Dichloromethane, ethanol and dimethylformamide (DMF) were supplied by Aibi Chemical Reagent Limited Company (Shanghai, China). Ultrapure water of $18 \mathrm{M} \Omega$ resistances was obtained from a Mingche D-24 ${ }^{\mathrm{UV}}$ purification device (Millipore, France). Three kinds of environmental water samples were collected from ChanZhi Reservoir, YiFu Reservoir and ShiPeng Reservoir, respectively.

\subsection{Instruments for characterization}

PerkinElmer Frontier (PerkinElmer, USA) spectrometer with the wave number range from 4000 to $400 \mathrm{~cm}^{-1}$ was used to record the Fourier transform infrared (FT-IR) spectra. D8 Advance (Bruker, USA) was used to record the X-ray diffractometer (XRD) patterns. SUPRA 55 scanning electron microscope (SEM, ZEISS, Germany) was used to characterize the morphologies of MOFs materials. Thermogravimetric analyzer SDTQ 600 (TA, USA) system was heated from $50{ }^{\circ} \mathrm{C}$ to $800{ }^{\circ} \mathrm{C}$ at the rate of $10{ }^{\circ} \mathrm{C} \mathrm{min}^{-1}$ to conduct the thermogravimetric analysis (TGA). 3H-2000PS1 (BeiShiDe, China) was used to calculate the Brunauer-Emmet-Teller (BET) surface area by nitrogen adsorptiondesorption analysis. Zetasizer (ZetaPlus, Brookhaven) was used to analyze the Zeta potential change. Thermo SCIENTIFIC ESCALAB 250Xi was used to record X-Ray photoelectron spectroscopic (XPS) characterization. Bruker AVANCE III $600 \mathrm{HZ}$ was used to record nuclear magnetic resonance (NMR) characterization.

\subsection{Preparation of UiO-66- $\mathrm{NMe}_{3}{ }^{+}$}

First, UiO-66-amine was synthesized by using a typical solvothermal method (Massoudinejad et al., 2016). Briefly, $0.233 \mathrm{~g} \mathrm{ZrCl}_{4}$ and $0.161 \mathrm{~g}$ 2-amino-terephthalic acid were dissolved in $50 \mathrm{~mL} \mathrm{DMF}$, and the mixture solution was transferred to a Teflon-lined stainless steel autoclave $(100 \mathrm{~mL})$ and heated to $120{ }^{\circ} \mathrm{C}$ for $48 \mathrm{~h}$. After cooling naturally to room temperature, the resulting UiO-66-amine was collected by centrifugation and then washed with DMF and ethanol successively; the precipitate was further washed by ethanol using Soxhlet extraction at $80{ }^{\circ} \mathrm{C}$ for $24 \mathrm{~h}$ to remove residual 2-amino-terephthalic acid. The resulting UiO-66-amine was dried in vacuum oven at $70{ }^{\circ} \mathrm{C}$ for $12 \mathrm{~h}$.

Then, UiO-66- $\mathrm{NMe}_{3}$ was synthesized as follows. $60 \mathrm{mg}$ of UiO-66amine and $70 \mu \mathrm{L}$ of methyl triflate were dispersed in $6 \mathrm{~mL}$ of dichloromethane. The mixture solution was stirred for $12 \mathrm{~h}$ at room temperature. Dichloromethane and unreacted methyl triflate were removed by drying the product in vacuum. Subsequently, the resulting product was further washed by ethanol using Soxhlet extraction at $80{ }^{\circ} \mathrm{C}$ for $24 \mathrm{~h}$ to thoroughly remove the residual methyl triflate. Finally, the resulting UiO-66- $\mathrm{NMe}_{3}$ was dried in a vacuum oven at $70{ }^{\circ} \mathrm{C}$ for $12 \mathrm{~h}$.

The quaternary anionic exchangers were synthesized by treating the UiO-66- $\mathrm{NMe}_{3}{ }^{+}$with $0.1 \mathrm{M} \mathrm{HCl}$ for $12 \mathrm{~h}$ at room temperature. Then the acidified MOFs were washed repeatedly by ultrapure water until the $\mathrm{pH}$ of eluent reaching neutral. Finally, $\mathrm{Cl}^{-}$became the anion exchangers of the synthesized UiO-66- $\mathrm{NMe}_{3}{ }^{+}$by drying it in a vacuum oven at $80{ }^{\circ} \mathrm{C}$ for $12 \mathrm{~h}$. The synthesis procedure was schematically shown in Fig. 1. 

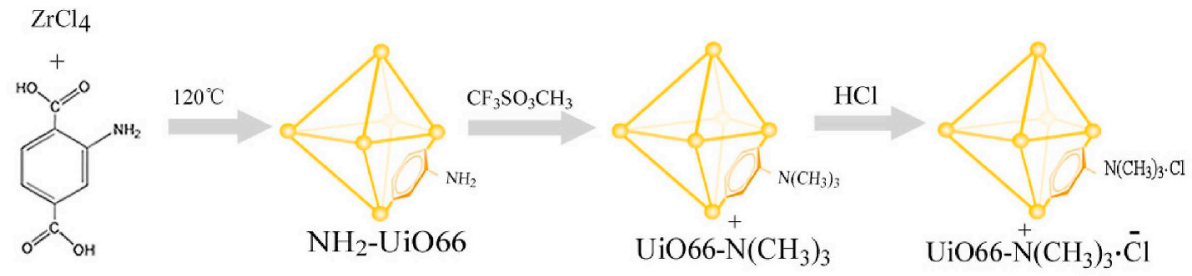

\subsection{Adsorption experiments}

The stock solution of 2,4-D at $1000 \mathrm{mg} \mathrm{L}^{-1}$ were prepared by dissolving solid standards in methanol. Daily used solutions were made from successive dilution of the stock solution with ultrapure water to appropriate concentrations. The concentrations of 2,4-D were analyzed by Ion Chromatography system (ThermoFisher Scientific Aquion) equipped with a Dionex AERS 500 suppressor (AERS $4 \mathrm{~mm}$ ) and an EGC eluent generator. An analytical column Dionex IonPacAS19 $(4 \times 250 \mathrm{~mm})$ was used at $30^{\circ} \mathrm{C}$. The mobile phase was $20 \mathrm{mM} \mathrm{NaOH}$ aqueous solution produced by eluent generator.

The batch adsorption experiment was conducted using a series of $250 \mathrm{~mL}$ conical flasks with a certain account of adsorbents and $20 \mathrm{~mL}$ of 2,4-D solution, and the flasks were placed in a constant temperature shaker for a fixed time. After adsorption, the adsorbents were separated by centrifuge from aqueous phase, and the solution was filtered with a $0.22 \mu \mathrm{m}$ nylon membrane. Then, remaining concentration of 2,4-D in filtrate was measured by ion chromatography. The adsorption capacity $\mathrm{q}_{\mathrm{e}}\left(\mathrm{mg} \mathrm{g}^{-1}\right)$ was expressed by Eq. (1):

$q_{\mathrm{e}}=\frac{\left(C_{0}-C_{\mathrm{e}}\right) V}{W}$

where $C_{0}$ and $C_{\mathrm{e}}\left(\mathrm{mg} \mathrm{L}^{-1}\right)$ mean the liquid-phase concentrations of 2,4Dat initial and equilibrium, respectively. $W(\mathrm{~g})$ is the mass of the adsorbent, and $V(\mathrm{~L})$ is the volume of solution.

\section{Results and discussion}

\subsection{Characterization of the adsorbent}

Fig. 2A shows the FT-IR spectra of UiO-66 (a), UiO-66-amine (b) and UiO-66- $\mathrm{NMe}_{3}{ }^{+}$(c). The bands at $1420 \mathrm{~cm}^{-1}$ and $1655 \mathrm{~cm}^{-1}$ were attributed to -O-C-O- groups and the bands at $1385 \mathrm{~cm}^{-1}$ and $1578 \mathrm{~cm}^{-1}$ indicated $\mathrm{C}=\mathrm{C}$ stretching vibration, revealing the terephthalic acid ligands were incorporated in the UiO-66 framework. Compared to the absorption bands of UiO-66, the bands at $1250 \mathrm{~cm}^{-1}$ and $965 \mathrm{~cm}^{-1}$ in Fig. 2A (b) may be attributed to the $\mathrm{C}-\mathrm{N}$ stretching vibration, and the band at $3400 \mathrm{~cm}^{-1}$ could be related to $-\mathrm{NH}_{2}$ stretching vibration and regarded as the characteristic peaks of pure amine groups, demonstrating the existence of amino terephthalic acid in UiO-66-amine. As shown in Fig. 2A (c), the band indicating the C-N stretching vibration at $965 \mathrm{~cm}^{-1}$ was shifted to $1032 \mathrm{~cm}^{-1}$ in UiO-66- $\mathrm{NMe}_{3}{ }^{+}$, suggesting the formation of quaternary ammonium groups in the synthesized material (Makvandi et al., 2016). Compared to the C-N stretching vibration in UiO-66-amine at $1250 \mathrm{~cm}^{-1}$, the band at $1280 \mathrm{~cm}^{-1}$ indicated a new type of $\mathrm{C}-\mathrm{N}$ stretching vibration existed in UiO-66$\mathrm{NMe}_{3}{ }^{+}$, demonstrating the formation of quaternary ammonium groups. Also, as seen in Fig. 2A, the peak at $655 \mathrm{~cm}^{-1}$ could be assigned to the $\mathrm{C}-\mathrm{H}$ bending vibration of benzene rings in terephthalic acid ligands (b), and then was shifted to a higher wavenumber of $670 \mathrm{~cm}^{-1}$ (c). The substituent group on benzene ring was modified from $-\mathrm{NH}_{2}$ to $-\mathrm{NMe}_{3}{ }^{+}$, and thereby the electron-donating effect was increased, leading to the peak shift of $\mathrm{C}-\mathrm{H}$ bending vibration. All the characteristic peaks indicated the successful preparation of quaternary amine anionic-exchange MOFs, namely UiO-66- $\mathrm{NMe}_{3}{ }^{+}$.

Fig. 2B shows the XRD patterns of UiO-66 (a), UiO-66-amine (b) and
Fig. 1. Schematic illustration of the synthetic process for $\mathrm{NMe}_{3}-\mathrm{UiO}-66$.
UiO-66- $\mathrm{NMe}_{3}{ }^{+}$(c). The diffraction peaks at $2 \theta=7.4,8.6$ and $25.8^{\circ}$ could be observed in three materials, which is consistent with that reported UiO-66 MOFs (Molavi et al., 2018). The result suggested that the three synthesized materials had the similar crystal structures and the introduction of quaternary ammonium groups did not cause significant changes in crystal structure of MOFs.

The morphologies of the obtained $\mathrm{UiO}-66-\mathrm{NMe}_{3}{ }^{+}$were characterized by SEM. As seen in Fig. S2, the unmodified UiO-66-amine showed a high crystallinity, and the surface of UiO-66-amine consisted of inter grown cubic nano-crystals (a and b), which are correspondent to previously reported UiO-66 MOFs (Yang et al., 2017b). The comparison of morphologies of UiO-66- $\mathrm{NMe}_{3}{ }^{+}$(c and d) suggested that the functionalization process did not change the crystallinity of MOF crystals. It should be noted the synthesized UiO-66-amine crystals were not uniformly dispersed, quite different from that reported (Zhang et al., 2016) following a similar method. This phenomenon was likely owing to that the synthesized UiO-66-amine crystals were washed with ethanol by Soxhlet extraction, and this procedure may well result in crystals conglutination and nonuniformity.

XPS characterization of UiO-66- $\mathrm{NMe}_{3}{ }^{+}$was carried out to define the chemical coordination environments within the MOF framework. Overall surveys of the prepared UiO-66- $\mathrm{NMe}_{3}{ }^{+}$were shown in Fig. $2 \mathrm{C}$ (I), and the C 1s (284.6 eV), Zr 3d (182.8 eV), O 1s (531.7 eV) N 1s (399.47) and Cl 2p (196.8) peaks appeared in the spectrum. The high resolution XPS spectrum for Zr 3d (Fig. 2C (II)) showed the double binding energy position of the $\mathrm{Zr} 3 \mathrm{~d} 3 / 2$ and $\mathrm{Zr} 3 \mathrm{~d} 5 / 2$ peaks were around 185.0 and $182.7 \mathrm{eV}$, respectively. The data are consistent with the reported values of UiO-66 (Yang et al., 2019). The binding peaks of C 1s (Fig. 2C (III)) at 288.5, 285.4 and $284.5 \mathrm{eV}$ were corresponding to carbon atoms in different functional groups such as $\mathrm{C}-\mathrm{O}, \mathrm{C}-\mathrm{NMe}_{3}$ and C-C (Liu et al., 2015). Additionally, the N 1s XPS spectrum (Fig. 2C (IV)) showed the binding energies at 399.0 and $400.4 \mathrm{eV}$, indicating the nitrogen atoms in $\mathrm{Ph}-\mathrm{NMe}_{3}$ and $\mathrm{C}-\mathrm{N}$ groups (Liu et al., 2015; Xu et al., 2013).

To further confirm the functionalization of quaternary amine groups, UiO-66-amine and $\mathrm{UiO}-66-\mathrm{NMe}_{3}{ }^{+}$were digested in acidic conditions ( $\mathrm{HF}$ in $\mathrm{d}_{6}$-DMSO) to obtain NMR data. As the $1 \mathrm{H}$ NMR spectrum of UiO-66-amine depicted in Fig. 3a, proton peaks at 7.05, 7.39 and $7.76 \mathrm{ppm}$ were attributed to 2 -aminoterephthalate ligand (Molavi et al., 2018; Goh et al., 2015). The common product of methylation of primary amine is a mixture of secondary, tertiary and quaternary amine products (Lebleu et al., 2014), and therefore, newly appearing proton peaks $(\delta=7.09$ for secondary amine, $\delta=7.10$ for tertiary amine and $\delta=7.22$ for quaternary amine (Lebleu et al., 2014) of UiO-66- $\mathrm{NMe}_{3}{ }^{+}$in Fig. 3b suggested the successful functionalization of UiO-66-amine. In addition, the functionalization degree of quaternary amine groups was about $55 \%$, which is calculated from the peak area proportion of quaternary amine in total newly appearing proton peaks for secondary amine, tertiary amine and quaternary amine, by referring to the related report (Lebleu et al., 2014).

The thermal stability analysis of the functionalized material was conducted with the temperature heating from 50 to $800{ }^{\circ} \mathrm{C}$. Fig. S3 shows the TGA curves of UiO-66 (a), UiO-66-amine (b) and UiO-66$\mathrm{NMe}_{3}{ }^{+}$(c). The decomposition processes of three materials were all mainly divided into three regions, and the TGA curves possessed the similar tendency. The first step weight-loss occurring around $120{ }^{\circ} \mathrm{C}$ 

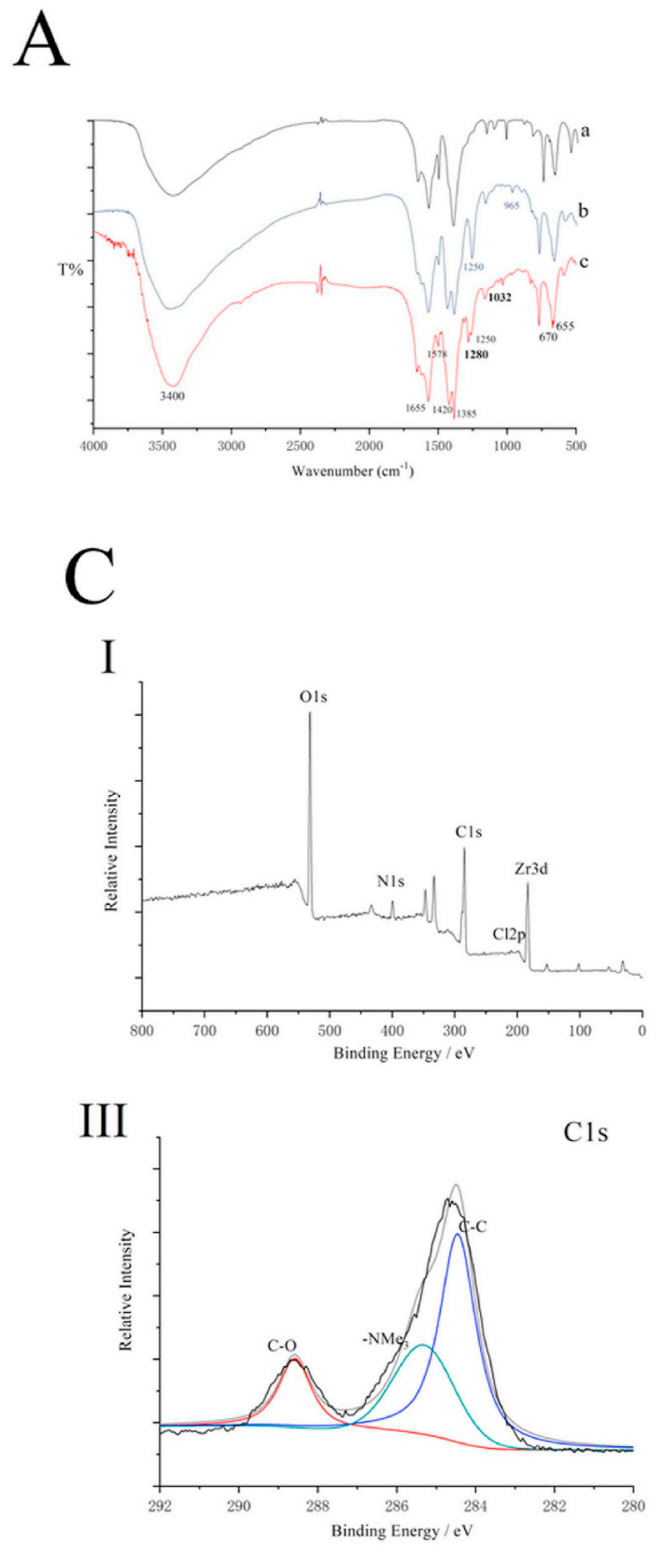

$\mathrm{B}$
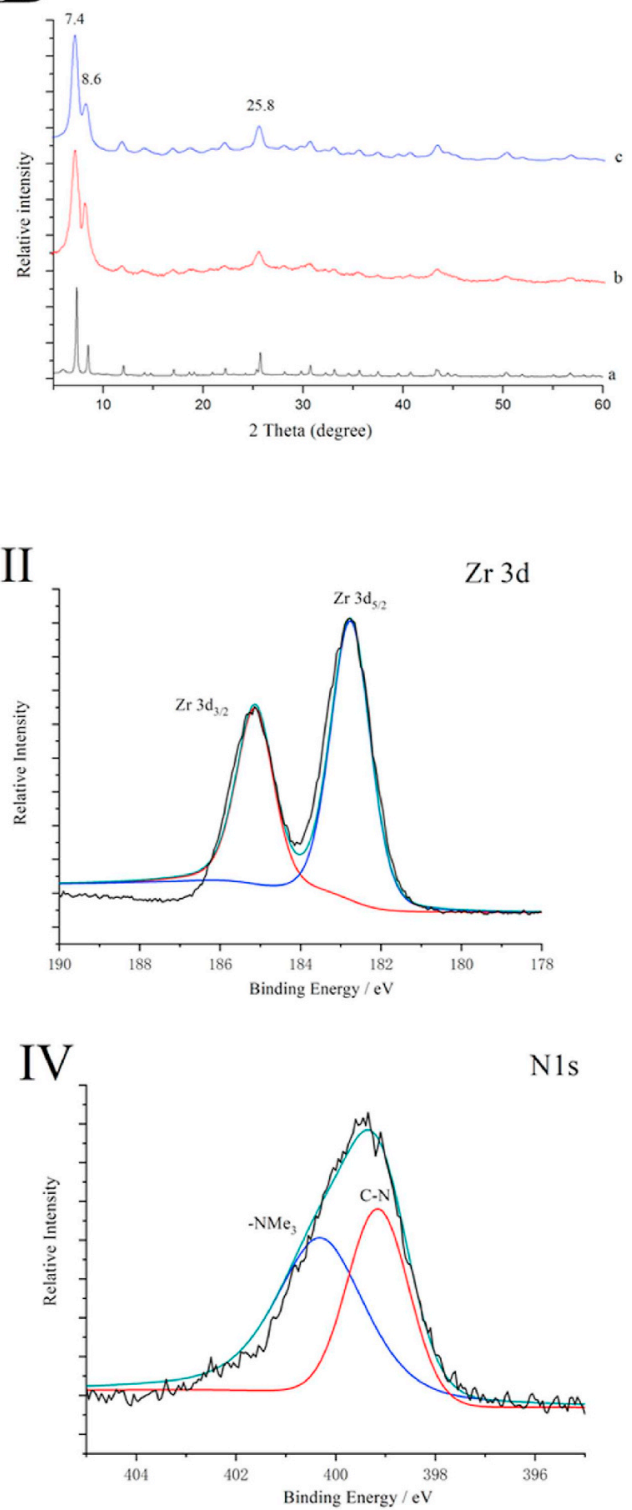

Fig. 2. (A) FT-IR spectra of UiO-66 (a), UiO-66- $\mathrm{NH}_{2}$ (b) and UiO-66- $\mathrm{NMe}_{3}{ }^{+}$(c). (B) XRD curves of UiO-66 (a), UiO-66-NH $\mathrm{N}_{2}$ (b) and UiO-66-NMe ${ }_{3}^{+}$(c). (C) Survey XPS spectra (I), and high-resolution spectra for Zr 3d (II), C1s (III) and N 1s (IV).

might be owing to the evaporation of water molecules, whereas the second weight loss from 120 to about $280^{\circ} \mathrm{C}$ might be due to the release of residual solvent from the pores of the framework. Subsequently, major weight losses appeared from 250 to $500{ }^{\circ} \mathrm{C}$ could be attributed to the decomposition of organic linkers of the framework. As the temperature was over $500{ }^{\circ} \mathrm{C}$, the weight of three materials all reduced slightly until $800{ }^{\circ} \mathrm{C}$, suggesting the formation of zirconium oxides derived from UiO based MOFs (Lin et al., 2016). Because UiO-66$\mathrm{NMe}_{3}{ }^{+}$comprised additional quaternary ammonium groups, organic matters possessed higher ratio in the framework. Thus, the remanent weight of UiO-66- $\mathrm{NMe}_{3}{ }^{+}$was lower than that of UiO-66-amineand UiO-66.

The BET surface area was investigated by nitrogen adsorption-desorption test. Fig. S4 shows the related isothermal curves of UiO-66 (a), UiO-66-amine (b) and UiO-66- $\mathrm{NMe}_{3}{ }^{+}$(c). And the BET surface areas of three materials were 861,509 and $484 \mathrm{~m}^{2} \mathrm{~g}^{-1}$, respectively. As seen, the surface area of UiO-66-amine was lower than that of UiO-66, which might be explained that the introduction of amine groups made the internal void space of the framework decreased (Saleem et al., 2016). In the same way, the reduced BET surface area of UiO-66- $\mathrm{NMe}_{3}{ }^{+}$compared to UiO-66-amine can be attributed to the formation of quaternary ammonium groups. The type IV isotherms for UiO-66- $\mathrm{NH}_{2}$ and UiO-66$\mathrm{NMe}_{3}{ }^{+}$materials indicated the existence of mesoporous, which could be attributed to the interparticle separations (Das et al., 2011; Bhunia et al., 2014). The presence of hysteresis loop might be due to the capillary condensation of these two materials and the introduction of functional groups of $-\mathrm{NH}_{2}$ and $-\mathrm{NMe}_{3}$ enlarged the pore size. Pore size distribution plots using the density functional theory (DFT) were shown inside Fig. S4. The sharpest peak of UiO-66, UiO-66- $\mathrm{NH}_{2}$ and UiO-66$\mathrm{NMe}_{3}{ }^{+}$occurred at pore diameter about $0.47,0.49,0.51 \mathrm{~nm}$, respectively. The size of 2,4-D is about $8.8 \AA$, which is a bit larger than the average pore size of UiO-66- $\mathrm{NMe}_{3}{ }^{+}$, and therefore, adsorption would primarily occur on the exposed surface of MOFs. On the other hand, as pore size distribution plots shown, some pores larger than $8.8 \AA$ also exist in the UiO-66- $\mathrm{NMe}_{3}{ }^{+}$materials (Zhang et al., 2019), which could make the interior adsorption sites interact with 2,4-D molecules, leading to the increase of adsorption capability. 


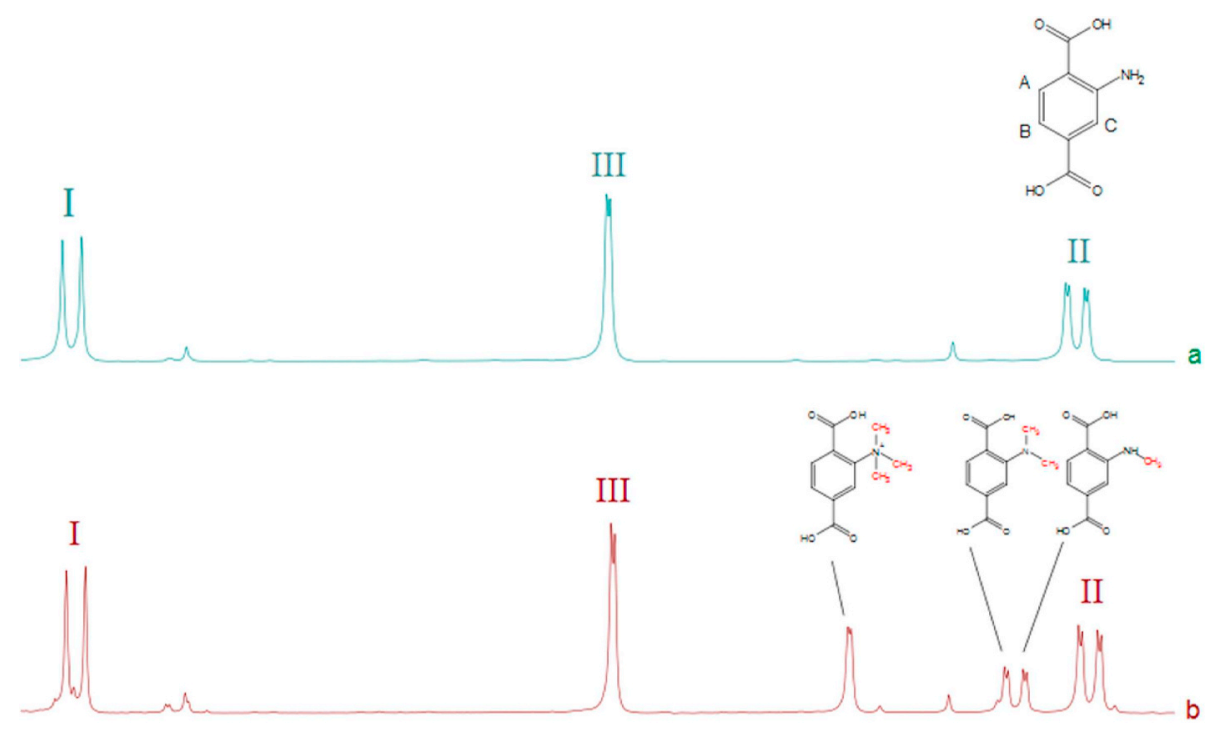

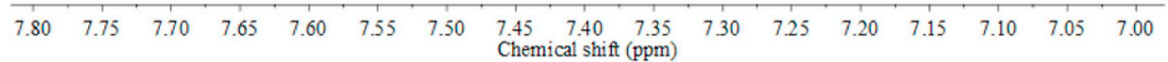

Fig. 3. $1 \mathrm{H}$ NMR spectrum of $\mathrm{UiO}-66-\mathrm{NH}_{2}$ (a) and UiO-66- $\mathrm{NMe}_{3}{ }^{+}$(b).

\subsection{Influence factors examination on adsorption}

\subsubsection{Effect of adsorbent dosage}

As Eq. (1) suggested, the adsorption capacity $\left(\mathrm{q}_{\mathrm{e}}\right)$ was directly affected by the amount of adsorbents. Therefore, 1-5 mg of adsorbents wereput into a series of $20 \mathrm{~mL}$ of $2,4-\mathrm{D}\left(10 \mathrm{mg} \mathrm{L}^{-1}\right)$ solutions to investigate the effect of adsorbent dosage. The adsorption capacities of three materials all kept decreasing when the amount of adsorbents increased from 1 to $5 \mathrm{mg}$, and the decrease tendencies were not linear, as the left ordinate shown in Fig. S5. This phenomenon might result from that at lower adsorbent dosage, the fixed amount of adsorption sites could be exposed with more 2,4-D molecules, leading to a higher adsorption capacity. Meanwhile, the relationship of removal efficiencies with the adsorbent dosage was also investigated. As the right ordinate shown in Fig. S5, the removal efficiencies sharply increased and then tended to be gentle within the tested range of adsorbent dosage. More 2,4-D molecules in water could be adsorbed by higher amount of adsorbents, resulting in the increase of removal efficiency. Therefore, compromising the adsorption capacities with the removal efficiencies, the dosage of $2 \mathrm{mg}$ of adsorbent was selected for further experiment.

\subsubsection{Effect of initial solution $p H$}

The $\mathrm{pH}$ value of the solution is an important variable throughout the adsorption process, which affects not only the degree of ionization of the polar adsorbates but also the surface charge of the adsorbent. In this procedure, the solution $\mathrm{pH}$ was evaluated ranging from 2.0 to 10.0 by using $0.01 \mathrm{~mol} \mathrm{~L}^{-1}$ of $\mathrm{HCl}$ or $\mathrm{NaOH}$ for adjustment, with the initial 2,4D concentration at $10 \mathrm{mg} \mathrm{L}^{-1}$. The $\mathrm{pKa}$ of 2,4-D is about 2.73 (PPDB, 2006; Njoku et al., 2015), as a result, the molecules will predominately exist in anionic species when $\mathrm{pH}>3$. As shown in Fig. 4, at $\mathrm{pH}$ of 2 , the highest adsorption capacity was attained by the UiO-66- $\mathrm{NMe}_{3}{ }^{+}$. Cation- $\pi$ boning may well appear between the natural form 2,4-D and cationic quaternary amine group in $\mathrm{UiO}-66-\mathrm{NMe}_{3}{ }^{+}$, since cation- $\pi$ boning can be formed between the cationic group and $\pi$-electron-rich aromatic structure (Zhao et al., 2017). On the other hand, the pure UiO66 also exhibited certain adsorption capability toward 2,4-D at the $\mathrm{pH}$ of 2 (Fig. 4), considering that there were no cationic groups on pure UiO- 66 , thus $\pi-\pi$ conjugation was very likely to happen between terephthalic acid in the framework of UiO-66 and 2,4-D. With the $\mathrm{pH}$ values increasing, 2,4-D molecules became deprotonated and the

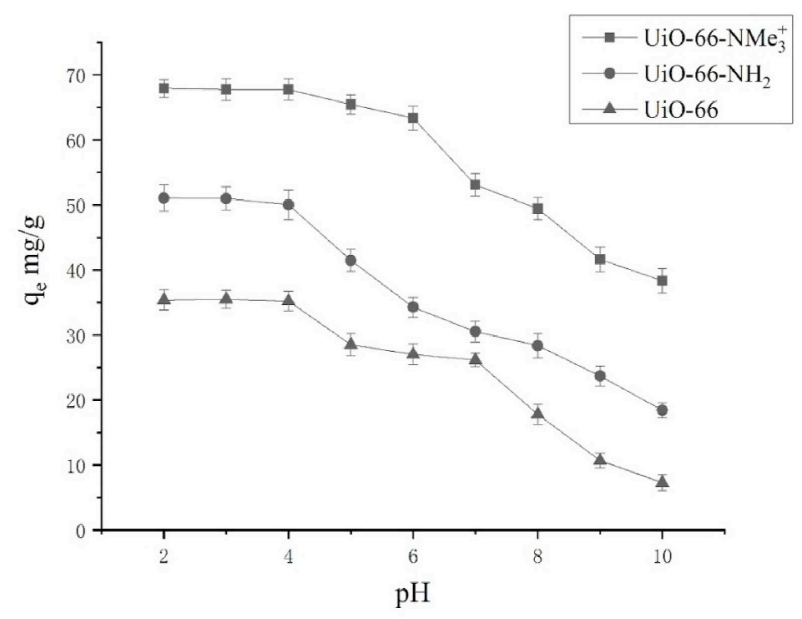

Fig. 4. Effect of solution $\mathrm{pH}$ values on the adsorption of 2,4-D by series UiO-66 materials. Experimental conditions: initial concentration, $10 \mathrm{mg} \mathrm{L}^{-1}$; adsorbent dosage, $2 \mathrm{mg}$; adsorption time, $120 \mathrm{~min}$; salt, without addition.

electron density in benzene ring was enhanced while $\pi-\pi$ conjugations were reduced. As the zeta potentials shown in Fig. S6, three kinds of MOFs were positively charged when $\mathrm{pH}$ values were $2-4$, and electrostatic interactions were increased when 2,4-D molecules became deprotonated; consequently, the adsorption capacities were almost unchanged at this $\mathrm{pH}$ range. The higher adsorption capacity of quaternized MOF suggested the electrostatic interactions significantly enhanced the adsorption of 2,4-D onto UiO-66- $\mathrm{NMe}_{3}{ }^{+}$. Moreover, with the solution $\mathrm{pH}$ value continuously increasing from 4-10, more anionic $\mathrm{OH}^{-}$existent in the solution would compete for the positive charged adsorption sites of $\mathrm{UiO}-66-\mathrm{NMe}_{3}{ }^{+}$, leading to the adsorption capacities of 2,4-D decreasing. Therefore, the main adsorption mechanism of UiO66- $\mathrm{NMe}_{3}{ }^{+}$towards 2,4-D can be proposed as a combination of electrostatic interactions, cation- $\pi$ boning and $\pi-\pi$ conjugations, and the ion exchange sites on UiO-66- $\mathrm{NMe}_{3}{ }^{+}$significantly enhanced adsorption capabilities for 2,4-D. 


\subsubsection{Effect of coexistent anions}

Coexistent inorganic anions in natural water bodies might competitively occupy the adsorption sites of the quaternary anionic exchange adsorbent. Therefore, the effects of different types of anions (sulfate, chloride and hydroxyl) on 2,4-D removal were investigated. In these batch experiments, equivalent concentrations of each coexistent anion were fixed as $1 \mathrm{meq} \mathrm{L}^{-1}$ with the concentration of 2,4-D solutions at $10 \mathrm{mg} \mathrm{L}^{-1}$. The comparison results of effect degree for each type of anion were presented in Fig. S7. As shown in the figure, the removal efficiency of 2,4-D was 72.5\% when no other anion was present in 2,4-D solution. However, the removal efficiencies of 2,4-D decreased to $40.4 \%, 39.3 \%$, and $67.2 \%$ when chloride, hydroxyl and sulfate were dosed, respectively. The monovalent ions (chloride, hydroxyl) had larger negative effect than divalent ion (sulfate). The smaller chloride ions would quickly occupy the adsorption sites of UiO-66- $\mathrm{NMe}_{3}{ }^{+}$, and thereby significantly impede the adsorption of 2,4-D molecules. However, the sulfate is relatively harder to rapidly access to the quaternary amine groups of UiO-66- $\mathrm{NMe}_{3}{ }^{+}$due to their tetrahedral structure, and therefore, the interactions between 2,4-D molecules and UiO-66$\mathrm{NMe}_{3}{ }^{+}$was slightly impeded by sulfate (Wang et al., 2016a).

\subsection{Adsorption properties and models}

\subsubsection{Adsorption kinetics}

Adsorption kinetics is often used to describe the adsorption reaction rate and predict the adsorption mechanism. Three curves of adsorption capacities at initial concentration as $20 \mathrm{mg} \mathrm{L}^{-1}$ for UiO-66, UiO-66amine and $\mathrm{UiO}-66-\mathrm{NMe}_{3}{ }^{+}$vs. time were shown in Fig. 5A. The
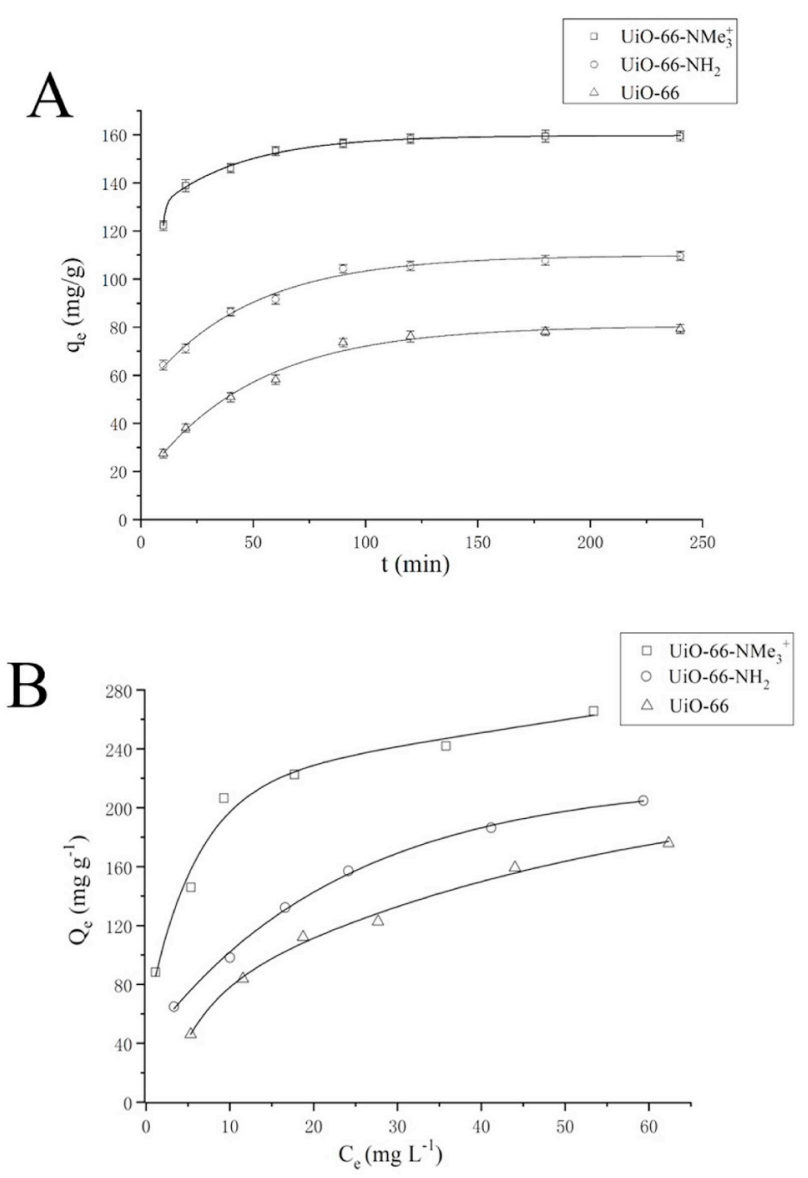

Fig. 5. (A) Effect of contact time on the adsorption of 2,4-D by series UiO-66 materials. Experimental conditions: initial concentration, $20 \mathrm{mg} \mathrm{L}^{-1}$; adsorbent dosage, $2 \mathrm{mg}$; pH, without adjusting; salt: without addition. (B) Adsorption isotherms of $2,4-\mathrm{D}$ by series UiO-66 materials at $25{ }^{\circ} \mathrm{C}$.
Table 1

Kinetic model parameters of 2,4-D adsorption onto series UiO-66 materials.

\begin{tabular}{lllllllll}
\hline Adsorbent & $\begin{array}{l}\mathrm{q}_{\mathrm{e}} \\
(\mathrm{mg}\end{array}$ & \multicolumn{3}{l}{ Pseudo-first-order kinetics } & \multicolumn{3}{l}{ Pseudo-second-order kinetics } \\
\cline { 3 - 9 } $\left.\mathrm{g}^{-1}\right)$ & $\mathrm{k}_{1}\left(\mathrm{~min}^{-1}\right)$ & $\begin{array}{l}\mathrm{q}_{\mathrm{e}, \mathrm{cal}} \\
(\mathrm{mg} \\
\left.\mathrm{g}^{-1}\right)\end{array}$ & $\mathrm{R}^{2}$ & $\begin{array}{l}\mathrm{K}_{2}\left(\mathrm{~g} \mathrm{mg}^{-1}\right. \\
\left.\mathrm{min}^{-1}\right)\end{array}$ & $\begin{array}{l}\mathrm{q}_{\mathrm{e}, \mathrm{cal}} \\
(\mathrm{mg}\end{array}$ & $\mathrm{R}^{2}$ \\
& & & & & $\left.\mathrm{~g}^{-1}\right)$ & \\
\hline UiO-66- $\mathrm{NMe}_{3}{ }^{+}$ & 160 & 0.03 & 45 & 0.992 & $1.7 \times 10^{-3}$ & 162 & 0.999 \\
UiO-66 $-\mathrm{NH}_{2}$ & 110 & 0.023 & 60 & 0.974 & $0.6 \times 10^{-3}$ & 125 & 0.999 \\
UiO-66 & 80 & 0.026 & 74 & 0.973 & $0.4 \times 10^{-3}$ & 91 & 0.996 \\
\hline
\end{tabular}

adsorption capacities of three tested materials increased with a similar trend, and all achieved equilibrium at about $120 \mathrm{~min}$. Therefore, the adsorption process can reach equilibrium at about $120 \mathrm{~min}$ and this time was chosen for the following experiments.

The diffusion rate of the adsorption process was estimated by two kinds of kinetic models, namely the pseudo first-order (Eq. (2)) and pseudo second-order (Eq. (3)) models. Relevant equations and discussions were described in ESI.

Based on the slope and intercept of the above equations, the adsorption rate constant $\left(k_{1}, k_{2}\right)$ and calculated $q_{\mathrm{e}}$ values were displayed in Table 1 . The correlation coefficient $\left(R^{2}\right)$ values obtained from the pseudo-first-order model were $0.992,0.974$ and 0.973 for UiO-66$\mathrm{NMe}_{3}{ }^{+}$, UiO-66-amine and UiO-66, respectively. Whereas for the pseudo-second-order model, $R^{2}$ values were $0.999,0.999$ and 0.996 for UiO-66- $\mathrm{NMe}_{3}{ }^{+}$, UiO-66-amine and UiO-66, respectively, and all sorption kinetics can be concluded to follow the pseudo-second-order model. Additionally, as shown in Table 1, the pseudo-second-order model showed better agreement between the experimental adsorption capacities ( $\left.q_{\mathrm{e}, \text { exp }}\right)$ with the calculated data $\left(q_{\mathrm{e}, \mathrm{cal}}\right)$, further revealing the better suitability of pseudo-second-order model in fitting the kinetic data. These results meant that the adsorption process should be more relying on chemical sorption; high correlation coefficients $\left(R^{2}\right)$ implied possible chemical interactions between the function groups on MOFs and 2,4-D. The 2,4-D molecules predominately existed in anionic species and a fraction natural forms under the tested conditions. Therefore, electrostatic interactions between anionic 2,4-D and cationic sites on MOFs might be the major adsorption mechanism; in addition, $\pi-\pi$ conjugation between the benzene rings within MOFs and 2,4-D also played an important role. Hydroxyl groups on metal oxide clusters ( $\mathrm{Zr}-\mathrm{OH})$ existing in the unit cell of UiO-66 framework (Wang et al., 2015) provided electron donor (Lewis base) and the $\mathrm{Cl}$ atom on 2,4-D molecules possessed electronegativity (Lewis acid). Therefore, Lewis acid-base interactions might exist in the adsorbent-adsorbates system.

\subsubsection{Adsorption isotherms}

Adsorption isotherms are used to acquire a better understanding of the distribution of adsorbate between the adsorbent surface and the liquid phase at adsorption equilibrium, which are beneficial for further illustrating the adsorption behavior and comparing the adsorption performances of different adsorbents. The 2,4-D solutions with different initial concentrations $\left(10,20,40,60,80 \mathrm{mg} \mathrm{L}^{-1}\right)$ were prepared for the isotherm tests with the adsorption temperature maintained at $25{ }^{\circ} \mathrm{C}$, and the results were shown in Fig. 5B. Two classic isotherm models named Langmuir (Eq. (4)) and Freundlich (Eq. (5)) were used to describe the equilibrium data. Relevant equations and discussions were displayed in ESI.

As the parameters of two adsorption models presented in Table 2, higher linear correlation coefficients $\left(R^{2}\right)$ values suggested that Langmuir-type isotherms showed a better fitting to adsorption data of three materials. This result suggested that 2,4-D molecules were mostly monolayer adsorption on the surface of adsorbents. As for the calculated maximum adsorption capacities $\left(q_{\mathrm{m}}\right)$, the quaternized anionicexchange MOF (UiO-66- $\mathrm{NMe}_{3}{ }^{+}$) exhibited the higher value $\left(279 \mathrm{mg} \mathrm{g}^{-1}\right)$ than that of unmodified UiO-66- $\mathrm{NH}_{2}\left(222 \mathrm{mg} \mathrm{g}^{-1}\right)$ and 
Table 2

Isotherm parameters of 2,4-D adsorption onto series UiO-66 materials.

\begin{tabular}{|c|c|c|c|c|c|c|}
\hline \multirow[t]{2}{*}{ Adsorbent } & \multicolumn{3}{|c|}{ Langmuir } & \multicolumn{3}{|l|}{ Freundlich } \\
\hline & $\begin{array}{l}Q_{m}(m g \\
\left.g^{-1}\right)\end{array}$ & $\begin{array}{l}\mathrm{K}_{\mathrm{L}}(\mathrm{L} \\
\left.\mathrm{mg}^{-1}\right)\end{array}$ & $\mathrm{R}^{2}$ & $\begin{array}{l}\mathrm{K}\left(\mathrm{mg} \mathrm{g} \mathrm{g}^{-1}(\mathrm{~L}\right. \\
\left.\left.\mathrm{mg}^{-1}\right)^{1 / \mathrm{n}}\right)\end{array}$ & $\mathrm{n}$ & $\mathrm{R}^{2}$ \\
\hline $\begin{array}{l}\text { UiO-66 - } \\
\mathrm{NMe}_{3}+\end{array}$ & 279 & 0.086 & 0.996 & 92.1 & 3.50 & 0.943 \\
\hline UiO-66- $\mathrm{NH}_{2}$ & 222 & 0.091 & 0.998 & 40.3 & 2.49 & 0.981 \\
\hline UiO-66 & 179 & 0.078 & 0.998 & 28.6 & 2.38 & 0.964 \\
\hline
\end{tabular}

pure UiO-66 (179 $\mathrm{mg} \mathrm{g}^{-1}$ ); the UiO-66- $\mathrm{NMe}_{3}{ }^{+}$with the lowest BET surface area attained the highest adsorption capacity. Generally, specific surface area of an adsorbent has a significant influence on the adsorption performance; the larger the specific surface area is, the greater the adsorption capacity is (Lei et al., 2017). However, the general phenomenon will only occur when Van Der Waals interaction is the main adsorption mechanism (Sarker et al., 2018). Herein, the adsorption process of 2,4-D on UiO-66- $\mathrm{NMe}_{3}{ }^{+}$was mainly dominated by electrostatic interactions combining with $\pi-\pi$ conjugations, and therefore, the adsorption capacity wasn't affected by the lower surface area. The anion exchangers $\mathrm{Cl}^{-}$was detected in the adsorption solution, indicating the ion-exchange interaction existed in adsorption process of UiO-66- $\mathrm{NMe}_{3}{ }^{+}$. The anion exchange capacity of this UiO-66- $\mathrm{NMe}_{3}{ }^{+}$ was estimated by a reported method (Olorunyomi et al., 2018) to be 3.3 $\mathrm{meq} / \mathrm{g}$.

The dimensionless separation factors $\left(R_{\mathrm{L}}\right)$ described by Eq. (6) are related to the favorability of an adsorption behavior (Wang et al., 2016b). Relevant equations and discussions were displayed in ESI. As can be seen from Fig. S8, $R_{\mathrm{L}}$ values of UiO-66- $\mathrm{NMe}_{3}{ }^{+}$, UiO-66-amine and UiO- 66 were between 0 and 1, suggesting the adsorption conditions were favorable (Wang et al., 2016b).

On the other hand, Freundlich model assumes that the adsorption occurs as multilayers on heterogeneous surfaces of adsorbents. The linear correlation coefficients $\left(R^{2}\right)$ obtained from the Freundlich model for UiO-66- $\mathrm{NMe}_{3}{ }^{+}$, UiO-66-amine and UiO-66 were 0.943, 0.981 and 0.964 , respectively, indicating the adsorption performances also fitted the Freundlich model. The higher $R^{2}$ of UiO-66-amine and UiO-66 suggested the adsorption of 2,4-D onto these two materials may involve both monomolecular-layer and multilayer adsorption. However, adsorption behavior of 2,4-D onto $\mathrm{UiO}-66-\mathrm{NMe}_{3}{ }^{+}$was dominated by monomolecular-layer sorption, indicating chemisorption was the main mechanism. This phenomenon suggested that the quaternary amines groups on the surface of UiO-66- $\mathrm{NMe}_{3}{ }^{+}$made electrostatic interactions become the key mechanism of the adsorption process. In addition, as can be seen in Table 2, the Freundlich constant ( $n$ ) values were falling between 2 and 10, suggesting that the adsorption behaviors of 2,4-D on three materials are favorable (Erdem et al., 2004).

\subsubsection{Adsorption thermodynamics}

The thermodynamic parameters such as $\Delta \mathrm{G}$ (Gibbs free energy change, $\mathrm{kJ} \mathrm{mol}^{-1}$ ), $\Delta \mathrm{H}$ (enthalpy change, $\mathrm{kJ} \mathrm{mol}^{-1}$ ) and $\Delta \mathrm{S}$ (entropy change, $\mathrm{J} \mathrm{mol}^{-1} \mathrm{~K}^{-1}$ ) were investigated at three different temperatures of 298,308 and $318 \mathrm{~K}$ for $120 \mathrm{~min}$. The values of $\Delta \mathrm{G}$ were calculated from Eq. (7). Additionally, $\Delta \mathrm{H}$ and $\Delta \mathrm{S}$ were determined from the slope of and the intercept of van't Hoff plot (Eq. (8)) of $\ln K$ versus $1 / T$. Relevant equations and discussions were displayed in ESI.

The linear plots of $\ln K$ versus $1 / T$ were presented in Fig. S9. The calculated thermodynamic parameters were shown in Table 3. The negative $\Delta G$ values at all temperatures indicated the spontaneous adsorption of 2,4-D onto series UiO-66 MOFs and the adsorption conditions were favorable. The values of $\Delta G$ became more negative with temperature declining suggesting that the adsorption processes of 2,4-D were exothermic and more favorable at lower temperatures. The calculated $\Delta H$ values were $-43.94,-38.28$ and $-29.33 \mathrm{~kJ} \mathrm{~mol}^{-1}$ for UiO-66- $\mathrm{NMe}_{3}{ }^{+}$, UiO-66-amine and UiO-66, respectively. The negative changes of enthalpy revealed the 2,4-D adsorption process onto three materials were exothermic. The negative values of $\Delta S$ were -67.57 , -59.63 and $-32.36 \mathrm{~J} \mathrm{~mol}^{-1} \mathrm{~K}^{-1}$ for UiO-66- $\mathrm{NMe}_{3}{ }^{+}$, UiO-66-amine and UiO-66, respectively. The negative $\Delta S$ indicating the free energy at the solid-solution interface decreased within the adsorption process, possibly due to the adsorption mechanism of the 2,4-D was the cooperation of ion exchange interaction and $\pi-\pi$ conjugation. Thus, the number of adsorbed 2,4-D molecules was larger than that of the desorbed exchanger $\mathrm{Cl}^{-}$in the adsorbents, leading to the decrease of free energy at the solid-solution interface (Jung et al., 2013).

\subsection{Reusability of the UiO-66-NMe ${ }_{3}{ }^{+}$adsorbent}

To assess the stability and reusability of the prepared UiO-66$\mathrm{NMe}_{3}{ }^{+}$adsorbent, $1 \% \mathrm{NaCl} /$ methanol (30:70, v/v) was selected as regeneration solution to conduct the adsorption-desorption experiments. As shown in Fig. S10, the reusability efficiencies of the UiO-66$\mathrm{NMe}_{3}{ }^{+}$didn't decrease noticeably after seven successive cycles, indicating the UiO-66- $\mathrm{NMe}_{3}{ }^{+}$adsorbents performed well in stability and reusability. FT-IR spectra were used to compare regenerated material with initial UiO-66- $\mathrm{NMe}_{3}{ }^{+}$. As shown in Fig. S11, the bands of -O-C-Ogroups appearing at 1420 and $1655 \mathrm{~cm}^{-1}$ and the bands at 1385 and $1578 \mathrm{~cm}^{-1}$ related to $\mathrm{C}=\mathrm{C}$ stretching vibration, still existed in the reused UiO-66- $\mathrm{NMe}_{3}{ }^{+}$framework. The band at $1032 \mathrm{~cm}^{-1}$ related to quaternary amine groups, and the band at $1250,1280 \mathrm{~cm}^{-1}$ related to C-N stretching vibration in UiO-66- $\mathrm{NMe}_{3}{ }^{+}$, were also present in the regenerated adsorbents. These results confirmed that the chemical structure of the quaternary amine anionic-exchange materials was stable. Meanwhile, as shown in Fig. S12, the XRD diffraction peaks at $2 \theta=7.4,8.6$ and $25.8^{\circ}$ related to UiO-66 MOFs could also be observed in reused $\mathrm{NMe}_{3}$-UiO-66, indicating the adsorption process didn't change the crystal structure of $\mathrm{NMe}_{3}$-UiO-66 adsorbents.

\subsection{Application of the UiO-66- $\mathrm{NMe}_{3}{ }^{+}$adsorbent to environmental water samples}

Furthermore, the adsorption capacity and reusability of UiO-66$\mathrm{NMe}_{3}{ }^{+}$in three real environmental waters were evaluated. Relevant experimental data were listed in Table S1. As seen from the table, under the same experimental conditions such as the initial concentration at $10 \mathrm{mg} \mathrm{L}^{-1}$, the adsorption capabilities of UiO-66- $\mathrm{NMe}_{3}{ }^{+}$for 2,4-D in the reservoir water samples (63-67 $\mathrm{mg} \mathrm{g}^{-1}$ ) aren't markedly decreased, comparing with that in ultrapure water sample $\left(71 \mathrm{mg} \mathrm{g}^{-1}\right)$; the material applied in reservoir waters also could be recycled for at least five times. Therefore, the UiO-66- $\mathrm{NMe}_{3}{ }^{+}$adsorbent possessed good

Table 3

Thermodynamic parameters of 2,4-D adsorption onto series UiO-66 materials.

\begin{tabular}{|c|c|c|c|c|c|c|c|c|c|}
\hline \multirow[t]{2}{*}{$\mathrm{T}(\mathrm{K})$} & \multicolumn{3}{|l|}{$\Delta \mathrm{G}\left(\mathrm{KJmol}^{-1}\right)$} & \multicolumn{3}{|l|}{$\Delta \mathrm{H}\left(\mathrm{KJmol}^{-1}\right)$} & \multicolumn{3}{|l|}{$\Delta \mathrm{S}\left(\mathrm{Jmol}^{-1} \mathrm{~K}^{-1}\right)$} \\
\hline & UiO-66 $\mathrm{NMe}_{3}{ }^{+}$ & $\mathrm{UiO}-66-\mathrm{NH}_{2}$ & UiO-66 & $\mathrm{UiO}-66 \mathrm{NMe}_{3}{ }^{+}$ & $\mathrm{UiO}-66-\mathrm{NH}_{2}$ & UiO-66 & UiO-66 $\mathrm{NMe}_{3}{ }^{+}$ & $\mathrm{UiO}-66-\mathrm{NH}_{2}$ & UiO-66 \\
\hline 298 & -23.87 & -20.56 & -19.65 & -43.94 & -38.28 & -29.33 & -67.57 & -59.63 & -32.36 \\
\hline 308 & -22.98 & -19.85 & -19.45 & & & & & & \\
\hline 318 & -22.53 & -19.35 & -18.99 & & & & & & \\
\hline
\end{tabular}


Table 4

Comparison of adsorption parameters for 2,4-D adsorption removal onto quaternary amine anionic-exchange UiO-66-NMe ${ }_{3}{ }^{+}$.

\begin{tabular}{|c|c|c|c|c|c|}
\hline Adsorbent & Initial concentration $(\mathrm{mg} / \mathrm{L})$ & Adsorption capacity (mg/g) & Equilibrium time (h) & Reusability & Refs \\
\hline Magnetic graphene & 50 & 32 & 6 & 4 & Liu et al. (2016) \\
\hline Granular activated carbon & 300 & 182 & 10 & $\mathrm{n}$ & Salmaa et al. (2011) \\
\hline $\mathrm{H}_{3} \mathrm{PO}_{4}$-activated carbon & 400 & 261 & 6 & $\mathrm{n}$ & Njoku et al. (2015) \\
\hline Chitosan & 20 & 11 & 1 & $\mathrm{n}$ & Harmoudi et al. (2014) \\
\hline Magnetic ion-exchangeresin & 20 & 47 & 1.5 & $\mathrm{n}$ & Ding et al. (2012) \\
\hline Magnetic ion-exchangeresin & 20 & 61 & 3 & 10 & (Lu et al. (2015)) \\
\hline Neemoil-phenolic resin treated lignocellulosic jute & 20 & 39 & 2 & 5 & Manna et al. (2016) \\
\hline Lewatit VP OC 1163 & 50 & 117 & 20 & $\mathrm{n}$ & (Vergili and Barlas (2009)) \\
\hline $\mathrm{UiO}-66-\mathrm{NMe}_{3}{ }^{+}$ & 80 & 279 & 2 & 7 & This work \\
\hline
\end{tabular}

applicability in real environmental waters.

\subsection{Comparison of the adsorption performance with reported adsorbents for 2,4-D}

Here, five significant adsorption behavior parameters including initial solution concentrations, equilibrium time, adsorbent dosage, adsorption capacity and reusability are compared with those of other reported methods for 2,4-D removal, as listed in Table 4. Various synthesized carbon materials such as graphene (Liu et al., 2016), granular activated carbon (Salmaa et al., 2011), $\mathrm{H}_{3} \mathrm{PO}_{4}$ functionalized activated carbon (Njoku et al., 2015), chitosan (Harmoudi et al., 2014) and ion exchange resins (Ding et al., 2012; Liu et al., 2015; Manna et al., 2016; Vergili and Barlas, 2009) have been used for adsorption removal of 2,4D from water. Comparing with above materials, our prepared UiO-66$\mathrm{NMe}_{3}{ }^{+}$exhibit higher adsorption capability, shorter adsorption time and good reusability. Therefore, the UiO-66- $\mathrm{NMe}_{3}{ }^{+}$materials can be proposed to be an efficient, recyclable and environmentally friendly adsorbent to remove 2,4-D herbicide from water environment.

\section{Conclusions}

In the present work, novel quaternary amine anionic-exchange MOFs (UiO-66- $\mathrm{NMe}_{3}{ }^{+}$) were successfully prepared and employed as adsorbents to remove 2,4-D from aqueous solutions. The prepared UiO$66-\mathrm{NMe}_{3}{ }^{+}$material was well characterized and the optimized adsorption conditions were obtained. The sorption mechanisms can be concluded as the combination of electrostatic interactions and $\pi-\pi$ conjugations, and the ion exchange sites on UiO-66- $\mathrm{NMe}_{3}{ }^{+}$significantly enhanced the adsorption capability of 2,4-D. Furthermore, the pseudo second-order kinetics model was more consistent with the experimental data and the Langmuir isotherm model was better fitted for the equilibrium adsorption behavior. Therefore, the adsorption process of 2,4-D onto UiO-66- $\mathrm{NMe}_{3}{ }^{+}$mostly belonged to chemisorption and monomolecular-layer adsorption. The maximum adsorption capacity of UiO66- $\mathrm{NMe}_{3}{ }^{+}$was $279 \mathrm{mg} \mathrm{g}^{-1}$, which was much higher than that of the unfunctionalized UiO-66-amine and pristine UiO-66. Thermodynamic study including the changes of Gibbs free energy, enthalpy and entropy suggested that the adsorption of 2,4-D onto UiO-66- $\mathrm{NMe}_{3}{ }^{+}$was spontaneous and exothermic as well as entropy decrease. Comparing with traditional resins and carbon materials, the presented UiO-66- $\mathrm{NMe}_{3}{ }^{+}$ exhibited higher adsorption capability, shorter adsorption time and good reusability, indicating the potential applicability of iMOFs for the removal of polar contaminant from water samples.

\section{CRediT authorship contribution statement}

Gege Wu: Conceptualization, Methodology, Investigation, Writing original draft. Jiping Ma: Conceptualization, Methodology, Funding acquisition, Supervision, Project administration, Writing - review \& editing. Shuang Li: Data curation, Investigation. Shasha Wang: Methodology, Investigation. Bo Jiang: Methodology, Investigation.
Siyi Luo: Methodology, Software. Jinhua Li: Methodology, Writing review \& editing. Xiaoyan Wang: Validation, Formal analysis, Writing - review \& editing. Yafeng Guan: Methodology, Supervision. Lingxin Chen: Supervision, Software, Writing - review \& editing.

\section{Declaration of competing interest}

The authors declare that they have no known competing financial interests or personal relationships that could have appeared to influence the work reported in this paper.

\section{Acknowledgments}

This work was financially supported by the National Natural Science Foundation of China (21976099, 21876199, 21804010), the Natural Science Foundation of Shandong Province of China (ZR2019MB046), the Qingdao Science and Technology Program of China (17-3-3-77nsh), the Department of Science and Technology of Shandong Province of China (GG201709290055), the Science and Technology Development Plan of Yantai (2017ZH093), Research Initiation Fund of Binzhou Medical University (Grant No. BY2019KYQD39) and the Taishan Scholar Project Special Funding (ts20190962).

\section{Appendix A. Supplementary data}

Supplementary data to this article can be found online at https:// doi.org/10.1016/j.envres.2020.109542.

\section{References}

Aaron, J.J., Oturan, M.A., 2001. New photochemical and electrochemical methods for the degradation of pesticides in aqueous media-environmental applications. Turk. J. Chem. 25, 509-520.

Azha, M.R., Abid, H.R., Periasamy, V., Sun, H.Q., Tade, M.O., Wang, S.B., 2017. Adsorptive removal of antibiotic sulfonamide by UiO-66 and ZIF-67 for wastewater treatment. J. Colloid Interface Sci. 500, 88-95.

Banerjee, D., Xu, W.Q., Nie, Z., Johnson, L.E.V., Coghlan, C., Sushko, M.L., Kim, D. Schweiger, M.J., Kruger, A.A., Doonan, C.J., Thallapally, P.K., 2016. Zirconium-based metal - organic framework for removal of perrhenate from water. Inorg. Chem. 55, 8241-8243.

Bhunia, S., Chatterjee, N., Das, S., Saha, K.D., Bhaumik, A., 2014. Novel porous polyurea network showing aggregation induced white light emission, applications as biosensor and scaffold for drug delivery. ACS Appl. Mater. Interfaces 6, 22569-22576.

Burtch, N.C., Jasuja, H., Walton, K.S., 2014. Water stability and adsorption in metal organic frameworks. Chem. Rev. 114, 10575-10612.

Corwin, C.J., Summers, R.S., 2012. Controlling trace organic contaminants with GAC adsorption. J. Am. Water Works Assoc. 104, 36-47.

Cycon, M., Zmijowska, A., Seget, Z.P., 2011. Biodegradation Kinetics of 2,4-D by bacterial strains isolated from soil. Cent. Eur. J. Biol. 6, 188-198.

Das, S.K., Bhunia, M.K., Seikh, M.M., Dutta, S., Bhaumik, A., 2011. Highly porous Co(II)salicylate metal-organic framework: synthesis, characterization and magnetic properties. Dalton Trans. 40, 2932-2939.

De, T.M., Jonchiere, R., Pullumbi, P., Coudert, F.X., Fuchs, A.H., 2012. How can a hydrophobic MOF be water-unstable? Insight into the hydration mechanism of IRMOFs. ChemPhysChem 13, 3497-3503.

Derylo-Marczewska, A., Blachnio, M., Marczewski, A.W., Swiatkowski, A., Tarasiuk, B., 2010. Adsorption of selected herbicides from aqueous solutions on activated carbon. J. Therm. Anal. Calorim. 101, 785-794.

Dias, E.M., Camille, P., 2015. Towards the use of metal-organic frameworks for water reuse: a review of the recent advances in the field of organic pollutants removal and 
degradation and the next steps in the field. J. Mater. Chem. 3, 22484-22506.

Ding, L., Lu, X., Deng, H.P., Zhang, X.X., 2012. Adsorptive removal of 2,4Dichlorophenoxyacetic Acid (2,4-D) from aqueous solutions using MIEX resin. Ind. Eng. Chem. Res. 51, 11226-11235.

Echavia, G.R.M., Matzusawa, F., Negishi, N., 2009. Photocatalytic degradation of organophosphate and phosphonoglycine pesticides using $\mathrm{TiO}_{2}$ immobilized on silica gel. Chemosphere 76, 595-600.

Ehtash, M., Salaün, M.C.F., Dimitrov, K., Salaün, P., Saboni, A., 2014. Phenol removal from aqueous media by pertraction using vegetable oil as a liquid membrane. Chem. Eng. J. 250, 42-47.

Erdem, E., Karapinar, N., Donat, R., 2004. The removal of heavy metal cations by natural zeolites. J. Colloid Interface Sci. 280, 309-314.

Gao, J., Huang, C.H., Lin, Y.F., Tong, P., Zhang, L., 2016. In situ solvothermal synthesis of metal-organic framework coated fiber for highly sensitive solid-phase microextraction of polycyclic aromatic hydrocarbons. J. Chromatogr. A 1436, 1-8.

Goh, T.W., Xiao, C.X., Maligal-Ganesh, R.V., Li, X.L., Huang, W.Y., 2015. Utilizing mixedlinker zirconium based metal-organic frameworks to enhance the visible light photocatalytic oxidation of alcohol. Chem. Eng. Sci. 124, 45-51.

Harmoudi, H.E., Gaini, L.E., Daoud, E., Rhazi, M., Boughaleb, Y., Mhammedi, M.A.E., Zalas, A.M., Bakasse, M., 2014. Removal of 2,4-D from aqueous solutions by adsorption processes using two biopolymers: chitin and chitosan and their optical properties. Opt. Mater. 36, 1471-1477.

Hasan, Z., Jhung, S.H., 2015. Removal of hazardous organics from water using metalorganic frameworks (MOFs): plausible mechanisms for selective adsorptions. J Hazard Mater. 283, 329-339.

He, C.T., Tian, J.Y., Liu, S.Y., Ouyang, G.F., Zhang, J.P., Chen, X.M., 2013. A porous coordination framework for highly sensitive and selective solid-phase microextraction of non-polar volatile organic compounds. Chem. Sci. 4 356-351.

Hu, C., He, M., Chen, B.B., Zhong, C., Hu, B., 2014b. Sorptive extraction using polydimethylsiloxane/metal-organic framework coated stir bars coupled with high performance liquid chromatography-fluorescence detection for the determination of polycyclic aromatic hydrocarbons in environmental water samples. J. Chromatogr. A 1356, 45-53.

Hu, P., Morabito, J.V., Tsung, C.K., 2014a. Core-shell catalysts of metal nanoparticle core and metal-organic framework shell. ACS Catal. 4, 4409-4419.

Ignatowicz, K., 2009. Selection of sorbent for removing pesticides during water treatment. J. Hazard Mater. 169, 953-957.

Jung, B.K., Hasan, Z., Jhung, S.H., 2013. Adsorptive removal of 2,4-dichlorophenoxyacetic acid (2,4-D) from water with a metal-organic framework. Chem. Eng. J. 234, 99-105.

Katsumata, H., Kobayashi, T., Kaneco, S., Suzuki, T., Ohta, K., 2011. Degradation of linuron by ultrasound combined with photo-Fenton treatment. Chem. Eng. J. 166 $468-473$.

Kumar, P., Pournara, A., Kim, K.H., Bansal, V., Rapti, S., Manos, M.J., 2017. Metal-organic frameworks: challenges and opportunities for ion-exchange/sorption applications. Prog. Mater. Sci. 86, 25-74.

Lebleu, T., Ma, X.L., Maddaluno, J., Legros, J., 2014. Selective monomethylation of primary amines with simple electrophiles. Chem. Commun. 50, 1836-1838.

Lei, C.S., Zhu, X.F., Zhu, B.C., Jiang, C.J., Le, Y., Yu, J.G., 2017. Superb adsorption capacity of hierarchical calcined $\mathrm{Ni} / \mathrm{Mg} / \mathrm{Al}$ layered double hydroxides for Congo red and $\operatorname{Cr}(\mathrm{VI})$ ions. J. Hazard Mater. 321, 801-811.

Lin, K.Y.A., Liu, Y.T., Chen, S.Y., 2016. Adsorption of fluoride to UiO-66- $\mathrm{NH}_{2}$ in water: stability, kinetic, isotherm and thermodynamic studies. J. Colloid Interface Sci. 461, 79-87.

Liu, K., Zhang, S.Y., Hu, X.Y., Zhang, K.Y., Roy, A., Yu, G., 2015. Understanding the adsorption of PFOA on MIL-101(Cr)-based anionic-exchange metal-organic frameworks: comparing DFT calculations with aqueous sorption experiments. Environ. Sci. Technol. 49, 8657-8665.

Liu, W., Yang, Q., Yang, Z.L., Wang, W.J., 2016. Adsorption of 2,4-D on magnetic graphene and mechanism study. Colloid. Surface. Physicochem. Eng. Aspect. 509, 367-375.

Lu, P., Deng, D.Y., Ni, X.D., 2012. Application of NaClO-treated multiwalled carbon nanotubes as solid phase extraction sorbents for preconcentration of trace 2,4-dichlorophenoxyacetic acid in aqueous samples. J. Separ. Sci. 35, 2307-2312.

Lu, W.H., Li, J.H., Sheng, Y.Q., Zhang, X.S., You, J.M., Chen, L.X., 2017. One-pot synthesis of magnetic iron oxide nanoparticle-multiwalled carbon nanotube composites for enhanced removal of $\mathrm{Cr}(\mathrm{VI})$ from aqueous solution. J. Colloid Interface Sci. 505, 1134-1146.

Lu, X., Shao, Y.S., Gao, N.Y., Ding, L., 2015. Equilibrium, thermodynamic, and kinetic studies of the adsorption of 2,4-dichlorophenoxyacetic acid from aqueous solution by MIEX resin. J. Chem. Eng. Data 60, 1259-1269.

Ma, J.P., Wu, G.G., Li, S., Tan, W.Q., Wang, X.Y., Li, J.H., Chen, L.X., 2018. Magnetic solid-phase extraction of heterocyclic pesticides in environmental water samples using metal-organic frameworks coupled to high performance liquid chromatography determination. J. Chromatogr. A 1553, 57-66.

Ma, J.P., Yao, Z.D., Hou, L.W., Lu, W.H., Yang, Q.P., Li, J.H., Chen, L.X., 2016. Metal organic frameworks (MOFs) for magnetic solid-phase extraction of pyrazole/pyrrole pesticides in environmental water samples followed by HPLC-DAD determination. Talanta 161, 686-692.

Makvandi, P., Ghaemy, M., Mohseni, M., 2016. Synthesis and characterization of photocurable bis-quaternary ammonium dimethacrylate with antimicrobial activity for dental restoration materials. Eur. Polym. J. 74, 81-90.

Manna, S., Saha, P., Roy, D., Sen, R., Adhikari, B., 2016. Removal of 2,4-dichlorophenoxyacetic acid from aqueous medium using modified jute. J. Taiwan. Inst. Chem. Eng. 67, 292-299.

Mantilla, A., Tzompantzi, F., Fernandez, J.L., Gongora, J., Mendoza, G., Gomez, R., 2009. Photodegradation of 2,4-dichlorophenoxyacetic acid using ZnAlFe layered double hydroxides as photocatalysts. Catal. Today 148, 119-123.
Massoudinejad, M., Ghaderpoori, M., Shahsavani, A., Amini, M.M., 2016. Adsorption of fluoride over a metal organic framework UiO-66 functionalized with amine groups and optimization with response surface methodology. J. Mol. Liq. 221, 279-286.

Molavi, H., Eskandari, A., Shojaei, A., Mousavi, S.A., 2018. Enhancing CO2/N2 adsorption selectivity via post-synthetic modification of NH2-UiO-66(Zr). Microporous Mesoporous Mater. 257, 193-201.

Njoku, V.O., Hameed, B.H., 2011. Preparation and characterization of activated carbon from corncob by chemical activation with $\mathrm{H}_{3} \mathrm{PO}_{4}$ for 2,4-dichlorophenoxyacetic acid adsorption. Chem. Eng. J. 173, 391-399.

Njoku, V.O., Islam, M.A., Asif, M., Hameed, B.H., 2015. Adsorption of 2,4-dichlorophenoxyacetic acid by mesoporous activated carbon prepared from $\mathrm{H}_{3} \mathrm{PO}_{4}$. activated langsat empty fruit bunch. J. Environ. Manag. 154, 138-144.

Olorunyomi, J.F., Chan, K.Y., Gao, L., Voskanyan, A.A., Li, C.V., 2018. Direct synthesis of anion exchange polymer threaded in a metal-organic framework through in situ polymerization of an ionic liquid. Microporous Mesoporous Mater. 259, 255-263.

PPDB, 2006. Pesticide Properties Database Developed by the Agriculture \& Environment Research Unit (AERU). University of Hertfordshire, UK.

Rapti, S., Pournara, A., Sarma, D., Papadas, I.T., Armatas, G.S., Tsipis, A.C., Lazarides, T., Kanatzidis, M.G., Manos, M.J., 2016. Selective capture of hexavalent chromium from an anion exchange column of metal organic resin-alginic acid composite. Chem. Sci. 7, 2427-2438.

Rosi, N.L., Eckert, J., Eddaoudi, M., Vodak, D.T., Kim, J., O'keeffe, M., Yaghi, O.M., 2003. Hydrogen storage in microporous metal-organic frameworks. Science 300, 1127-1129.

Rostamnia, S., Alamgholiloo, H., Liu, X., 2016. Pd-grafted open metal site copperbenzene1,4-dicarboxylate metal organic frameworks (Cu-BDC MOF's) as promising interfacial catalysts for sustainable Suzuki coupling. J. Colloid Interface Sci. 469, 310-317.

Saleem, H., Rafique, U., Davies, R.P., 2016. Investigations on post-synthetically modified UiO-66- $\mathrm{NH}_{2}$ for the adsorptive removal of heavy metal ions from aqueous solution. Microporous Mesoporous Mater. 221, 238-244.

Salmaa, J.M., Njokua, V.O., Hameed, B.H., 2011. Adsorption of pesticides from aqueous solution onto banana stalk activated carbon. Chem. Eng. J. 174, 41-48.

Sarker, M., Song, J.Y., Jhung, S.H., 2018. Carboxylic-acid-functionalized UiO-66-NH2: a promising adsorbent for bothaqueous- and non-aqueous-phase adsorptions. Chem. Eng. J. 331, 124-131.

Vergili, I., Barlas, H., 2009. Removal of 2,4-D, MCPA and Metalaxyl from water using Lewatit VP OC 1163 as sorbent. Desalination 249, 1107-1114.

Wang, C.H., Liu, X.L., Chen, J.P., Li, K., 2015. Superior removal of arsenic from water with zirconium metal organic framework UiO-66. Sci. Rep. 5, 16613-16623.

Wang, J.Y., Li, S.P., Xin, K.L., Tao, T., 2016a. Equilibrium and kinetic modeling of iron adsorption and the effect by chloride, sulfate, and hydroxyl: evaluation of PVC-U drinking pipes. Environ. Sci. Pollut. Res. 23, 23902-23910.

Wang, T., Zhao, P., Lu, N., Chen, H.C., Zhang, C.L., Hou, X.H., 2016b. Facile fabrication of Fe304/MIL-101(Cr) for effective removal of acid red 1 and orange $\mathrm{G}$ from aqueous solution. Chem. Eng. J. 295, 403-441.

Wu, G.G., Ma, J.P., Li, S., Guan, J., Jiang, B., Wang, L.Y., Li, J.H., Wang, X.Y., Chen, L.X., 2018. Magnetic copper-based metal organic framework as an effective and recyclable adsorbent for removal of two fluoroquinolone antibiotics from aqueous solutions. J. Colloid Interface Sci. 528, 360-371.

Xie, L.J., Liu, S.Q., Han, Z.B., Jiang, R.F., Liu, H., Zhu, F., Zeng, F., Su, C.Y., Ouyang, G.F., 2015. Preparation and characterization of metal-organic framework MIL-101(Cr)coated solid-phase microextraction fiber. Anal. Chim. Acta 853, 303-310.

Xu, J., Cao, Z., Liu, X., Zhao, H., Xiao, X., Wu, J.P., Xu, X.H., Zhou, J.L., 2016. Preparation of functionalized Pd/Fe- $\mathrm{Fe}_{3} \mathrm{O}_{4} @$ MWCNTs nanomaterials for aqueous 2,4-dichlorophenol removal: interactions, influence factors, and kinetics. J. Hazard Mater. 317, 656-666.

Xu, X., Gao, B.Y., Tan, X., Zhang, X.X., Yue, D.T., Yue, Q.Y., 2013. Uptake of perchlorate from aqueous solutions by amine-crosslinked cotton stalk. Carbohydr. Polym. 98, $132-138$.

Yang, C.X., Ren, H.B., Yan, X.P., 2013. Fluorescent metal-organic framework MIL-53(Al) for highly selective and sensitive detection of $\mathrm{Fe}^{3+}$ in aqueous solution. Anal. Chem. 85, 7441-7446.

Yang, Q.P., Li, L.J., Tan, W.Q., Sun, Y.J., Wang, H.L., Ma, J.P., Zhao, X.B., 2017a. Exceptional high selectivity of hydrogen/methane separation on a phosphonatebased MOF membrane with exclusion of methane molecules. Chem. Commun. 53, 9797-9800.

Yang, Q.X., Zhao, Q.Q., Ren, S.S., Chen, Z.J., Zheng, H.G., 2017b. Assembly of Zr-MOF crystals onto magnetic beads as a highly adsorbent for recycling nitrophenol. Chem. Eng. J. 323, 74-83.

Yang, Z.B., Zhu, L., Chen, L.A., 2019. Selective adsorption and separation of dyes from aqueous solution by core-shell structured NH2-functionalized UiO-66 magnetic composites. J. Colloid Interface Sci. 539, 76-86.

Zhang, F.M., Zheng, S., Xiao, Q., Zhong, Y.J., Zhu, W.D., Lin, A., Samy, E.M., 2016. Synergetic catalysis of palladium nanoparticles encaged within amine-functionalized UiO-66 in the hydrodeoxygenation of vanillin in water. Green Chem. 18, 2900-2908.

Zhang, L.J., Jian, Y., Wang, J., He, C., Li, X.Z., Liu, T., Duan, C.Y., 2012. Post-modification of a MOF through a fluorescent-labeling technology for the selective sensing and adsorption of $\mathrm{Ag}^{+}$in aqueous solution. Dalton Trans. 41, 10153-10155.

Zhang, Q., Zhang, Y., Chen, J., Liu, Q., 2019. Hierarchical structure kaolinite nanospheres with remarkably enhanced adsorption properties for methylene blue. Nanoscale Res. Lett. 14, 104.

Zhang, S.N., Han, P.P., Xia, Y., 2017. Facile extraction of azide in sartan drugs using magnetized anion-exchange metal-organic frameworks prior to ion chromatography. J. Chromatogr. A 1514, 29-35.

Zhao, Q., Zhang, S.Y., Zhang, X.J., Lei, L., Ma, W., Ma, C.X., Song, L., Chen, J.W., Pan, B., Xing, B.S., 2017. Cation-Pi interaction: a Key force for sorption of fluoroquinolone antibiotics on pyrogenic carbonaceous materials. Environ. Sci. Technol. 51 (23), 13659-13667. 